\title{
Extending the scope of PARP inhibitors in ovarian cancer
}

\section{Jonathan A Ledermann}

During the last decade trials with inhibitors of oral PARP (poly-ADP ribose polymerase), a key enzyme involved in the repair of DNA damage have led to a major change in the treatment of advanced ovarian cancer. A key element of the success of this class of drug is deficiency of homologous recombination repair (HRD) of DNA double-strand breaks. This is best seen in BRCA-mutated tumours as functioning BRCA proteins have a major role in preserving the complex DNA repair pathway. However, other mechanisms apart from BRCA mutations can also result in HRD and consequently lead to a clinical benefit from PARP inhibitors ${ }^{1}$. Study of DDR (DNA damage response) and manipulation of the process is now recognised as an important area of research as is could lead to better cancer treatments ${ }^{2}$. Precise measurement of HRD is difficult, but in ovarian cancer, it is most closely correlated with tumour platinum-sensitivity. Tumours that become platinum-resistant, an eventual inevitable consequence of recurrence, rarely respond to PARP inhibitors.

Olaparib, niraparib and rucaparib are PARP inhibitors used to treat ovarian cancer. They are more active in tumours with a BRCA-mutation but are also licensed as maintenance therapy following a response to platinum-based therapy. In these patients who have varying degrees of HRD they prolong the time to disease progression. Most tumours eventually become resistant to PARP inhibitors although there is a small cohort who continue without disease progression for many years ${ }^{3}$.

Repair of DNA strand breaks is a complex process, involving multiple proteins and more than one repair pathway. Similarly, a wide range of mechanisms underlie tumour resistance to PARP inhibitors and a number of different strategies are being pursued to prevent or overcome it ${ }^{4}$. The approach examined by Konstatinopoulos and colleagues uses an inhibitor of PI3 Kinase (alpelisib) in combination with the PARP inhibitor, olaparib 5 . The rationale for this combination is that PI3 Kinase inhibition has been shown to lead to a downregulation of $\mathrm{BRCA} 1 / 2$ proteins, increasing the degree of $\mathrm{HRD}^{6}$. In the absence of competent repair pathways, cells become sensitised to PARP inhibitors. This group has previously tested the combination of olaparib and the VEGFR inhibitor, cediranib in combination and demonstrated a synergistic effect of the two molecularly targeted drugs, but this was in a population of patients with tumours likely to be sensitive to platinum-based drugs and therefore PARP inhibitors ${ }^{7}$. In the phase I study of alpelisib and olaparib nearly all tumours were deemed resistant or refractory to platinum-based drugs (26 out of 28 patients), and thus unlikely to benefit from a PARP inhibitor, particularly in the absence of a BRCA mutation. The combination of these oral drugs was not without toxicity, but hyperglycaemia, nausea and abnormalities in liver transaminase, the most common adverse effects were generally manageable. Although toxicity was the primary endpoint, the response rate to this combination was much higher than would be expected from either drug alone in this group of patients. Most patients had ovarian cancer ( 4 had breast cancer) and with the ovarian cancer expansion cohort, the authors reported a partial response rate of $35.7 \%$ ( 10 out of 28 patients) and a median response duration from the time of first 
response of 5.5 months (ranging from 0.5 to 13.1 months). Disease stabilisation was seen in $50 \%$ of patients.

It is estimated that about $50 \%$ of high-grade serous ovarian tumours have a degree of HRD and are likely to benefit from PARP inhibitors. Many tumours, however, will not respond either $a b$ initio, or after they become resistant to platinum-based drugs. Exploiting the DNA damage response (DDR) pathways, as in the case of PARP inhibitors requires a detailed knowledge of DDR mechanisms in cells ${ }^{8}$. Current approaches being explored include the use of inhibitors of other pathways, such as CHK1, alone in BRCA wild-type tumours resistant to platinum or in the case of Wee1, in combination with chemotherapy ${ }^{9,10}$. Whilst it remains important to develop new therapies for patients with platinum-resistant ovarian cancer unlikely to respond to PARP inhibitors, more research is needed to enhance the activity of PARP inhibitors in patients who would otherwise have only a short-term benefit. Similarly, the strategy of re-introducing a PARP inhibitor following progression on treatment by adding another molecularly targeted agent, such as a PI3 kinase inhibitor, is attractive as there is a growing number of patients who have become resistant to PARP inhibitors. Identification of targetable pathways and more rationally designed trials with DDR inhibitors are needed to extend the significant clinical benefits that have been seen using PARP inhibitors.

\section{References}

1. Lord CJ, Ashworth A. PARP inhibitors: Synthetic lethality in the clinic. Science (New York, NY) 2017; 355(6330): 1152-8.

2. Brandsma I, Fleuren EDG, Williamson CT, Lord CJ. Directing the use of DDR kinase inhibitors in cancer treatment. Expert Opinion on Investigational Drugs 2017; 26(12): 134155.

3. Mirza MR, Pignata S, Ledermann JA. Latest clinical evidence and further development of PARP inhibitors in ovarian cancer. Annals of Oncology 2018; 29(6): 1366-76. 4. D'Andrea AD. Mechanisms of PARP inhibitor sensitivity and resistance. DNA repair 2018; 71: 172-6.

5. Konstantinopoulos $P$, Barry W, Birrer M, et al. Oral Poly(ADP-Ribose) Polymerase (PARP) Inhibitor Olaparib and Oral alpha Specific PI3-Kinase Inhibitor Alpelisib (BYL719): A Phase 1b Dose-Escalation Study with Dose Expansion in Patients with Ovarian Cancer. The Lancet Oncology 2019.

6. Rehman FL, Lord CJ, Ashworth A. The promise of combining inhibition of PI3K and PARP as cancer therapy. Cancer Discovery 2012; 2(11): 982-4.

7. Liu JF, Barry WT, Birrer M, et al. Combination cediranib and olaparib versus olaparib alone for women with recurrent platinum-sensitive ovarian cancer: a randomised phase 2 study. The Lancet Oncology 2014; 15(11): 1207-14.

8. Brown JS, O'Carrigan B, Jackson SP, Yap TA. Targeting DNA Repair in Cancer: Beyond PARP Inhibitors. Cancer Discovery 2017; 7(1): 20-37. 
9. Lee J-M, Nair J, Zimmer A, et al. Prexasertib, a cell cycle checkpoint kinase 1 and 2 inhibitor, in BRCA wild-type recurrent high-grade serous ovarian cancer: a first-in-class proof-of-concept phase 2 study. The Lancet Oncology 2018; 19(2): 207-15.

10. Leijen S, van Geel RMJM, Sonke GS, et al. Phase II Study of WEE1 Inhibitor AZD1775 Plus Carboplatin in Patients With TP53-Mutated Ovarian Cancer Refractory or Resistant to First-Line Therapy Within 3 Months. Journal of Clinical Oncology 2016; 34(36): 4354-61. 